\title{
Síndrome de realimentação: fatores de risco e diagnóstico
}

\author{
Feedback syndrome: risk factors $\mathbf{x}$ diagnosis \\ Síndrome de retroalimentación: factores de riesgo x diagnóstico
}

Carla Vasconcelos Afonso Gomes

ORCID: https://orcid.org/0000-0002-1347-5406 Faculdades Unidas do Norte de Minas Gerais, Brasil

E-mail: carlavasconcelos.med@gmail.com

Christiane Silva Souza

ORCID: https://orcid.org/0000-0001-6375-5986 Faculdades de Saúde Ibituruna, Brasil

E-mail: christianesilvasouza@outlook.com

Karla Talita Santos Silva

ORCID: https://orcid.org/0000-0001-5636-1655

Faculdades Unidas do Norte de Minas Gerais, Brasil

E-mail: karlatalitassilva@gmail.com

Sylmara Corrêa Monteiro

ORCID: https://orcid.org/0000-0003-4546-336X Instituto Federal de Educação, Ciência e Tecnologia do Norte de Minas Gerais, Brasil

E-mail: scmenfermagem9@gmail.com

Jessica Fernanda Silva Peres

ORCID: https://orcid.org/0000-0003-2750-5132 Faculdades de Saúde Ibituruna, Brasil E-mail: jessicafsilvaenf@gmail.com

Ana Paula de Oliveira Nascimento Alves ORCID: https://orcid.org/0000-0003-2144-3671 Universidade Federal dos Vales do Jequitinhonha e Mucuri, Brasil

E-mail: anapaula.nascimento@funorte.edu.br

Flavianny de Jesus Muniz

ORCID: https://orcid.org/0000-0002-7971-7340

Faculdades Unidas do Norte de Minas Gerais, Brasil

E-mail: flaviannyjmunizenf@hotmail.com

Fernando Lucas Freitas Rocha

ORCID: https://orcid.org/0000-0003-2974-4866

Faculdades Unidas do Norte de Minas Gerais, Brasil

E-mail: fernandolucasdrocha.enf@gmail.com

Taysa Cristina Cardoso Freitas

ORCID: https://orcid.org/0000-0003-3133-935X

Universidade Estadual de Montes Claros, Brasil E-mail: taysaccfreitas@ hotmail.com

Icaro Kelvin Botelho Dias

ORCID: https://orcid.org/0000-0002-7941-1034 Faculdades Unidas do Norte de Minas Gerais, Brasil E-mail: icarodias2809@hotmail.com

Isabella Batista Vieira

ORCID: https://orcid.org/0000-0002-0284-2338

Faculdades Unidas do Norte de Minas Gerais, Brasil

E-mail:isabellabvieira@gmail.com

Andreia Correia

ORCID: https://orcid.org/0000-0002-8936-2459

Faculdades Unidas do Norte de Minas Gerais, Brasil

E-mail: andreia.correiaenf@ hotmail.com

Lorrayne Oliveira Dias Soares

ORCID: https://orcid.org/0000-0001 75434549

Faculdades Unidas do Norte de Minas Gerais, Brasil

E-mail: lorrayne.oliveira.enf@gmail.com

Anne Christine Alves Pereira

ORCID: https://orcid.org/0000-0003-1772-3750

Faculdades Unidas do Norte de Minas Gerais, Brasil

E-mail: annecalvespereira@gmail.com

Samara Monteiro Rodrigues

ORCID: https://orcid.org/0000-0001-6679-5983

Faculdades Unidas do Norte de Minas Gerais, Brasil

E-mail: samara.monteiroti@gmail.com 


\begin{abstract}
Resumo
Objetivou-se descrever a síndrome da retroalimentação com enfoque nos fatores de risco e diagnósticos. As complicações metabólicas decorrentes da realimentação após jejum prolongado podem levar a patologia clínica atualmente definida como síndrome da realimentação. Descrita inicialmente durante a Segunda Guerra Mundial, quando prisioneiros da guerra apresentaram falência cardíaca após reposição nutricional, a síndrome da realimentação é uma desordem hidroeletrolítica grave, potencialmente fatal, que ocorre após a reintrodução da dieta, seja por via oral, enteral ou parenteral. Múltiplos sistemas podem ser afetados, incluindo o cardiovascular, o respiratório, e hematológico, o músculo esquelético e o neurológico.
\end{abstract}

Palavras-chave: Síndrome da realimentação; Desnutrição energética; Desnutrição.

\begin{abstract}
The objective was to describe the feedback syndrome with a focus on risk factors and diagnoses. Metabolic complications resulting from refeeding after prolonged fasting can lead to clinical pathology currently defined as refeeding syndrome. Initially described during World War II, when prisoners of war experienced heart failure after nutritional replacement, the feedback syndrome is a severe, potentially fatal, electrolyte disorder that occurs after the reintroduction of the diet, whether orally, enterally, or parenterally. Multiple systems can be affected, including cardiovascular, respiratory, and hematologic, skeletal and neurological muscle.
\end{abstract}

Keywords: Refeeding Syndrome; Energy Malnutrition; Malnutrition.

\title{
Resumen
}

El objetivo fue describir el síndrome de retroalimentación con un enfoque en los factores de riesgo y diagnósticos. Las complicaciones metabólicas resultantes de la realimentación después de un ayuno prolongado pueden conducir a una patología clínica actualmente definida como síndrome de realimentación. Descrito inicialmente durante la Segunda Guerra Mundial, cuando los prisioneros de guerra experimentaron insuficiencia cardíaca después del reemplazo nutricional, el síndrome de retroalimentación es un trastorno electrolítico severo y potencialmente fatal que ocurre después de la reintroducción de la dieta, ya sea por vía oral, enteral o parenteral. Pueden verse afectados múltiples sistemas, incluidos el músculo cardiovascular, respiratorio y hematológico, esquelético y neurológico.

Palabras clave: Síndrome de retroalimentación; Desnutrición energética; Desnutrición.

\section{Introdução}

O jejum é conceituado como a ausência da ingestão de alimentos e de nutrientes por um período mínimo de 6 horas, sendo definido prolongado quando superior a 72 horas. O jejum prolongado ocorre com periodicidade em pacientes hospitalizados, como para preparo de exames diagnósticos ou em períodos pré-operatórios, podendo ocasionar alterações metabólicas e nutricionais, além de facilitar para o aumento da prevalência de desnutrição hospitalar (Levenson, \& Seifter 1985; Waitzberg, Caiaffa, \& Correia, 2001).

As complicações metabólicas resultantes da realimentação após jejum prolongado foram inicialmente descrita em sobreviventes dos campos de concentração e associadas a inanição grave, constituindo a patologia clínica atualmente definida como síndrome da realimentação (Schnitzker, Mattman, \& Bliss, 1951; Brozek, Chapman, \& Keys, 1948).

A síndrome de realimentação (SR) é definida como uma complicação hidroeletrolítica considerada grave, com potencial de morte, que acontece após o processo de reintrodução da dieta, por via oral ou até mesmo parenteral. Essa condição foi relatada em literatura médica há mais de 65 anos, porém ainda pouco reconhecida, caracteriza-se por um grupo de sinais e sintomas clínicos que ocorrem em pacientes subnutridos e caquéticos realimentados após jejum prolongado (Mallet, 2002; Boateng, \& Sriram, 2010).

Pioneiramente foi descrita durante a Segunda Guerra Mundial, quando os indivíduos que eram prisioneiros do confronto apresentaram colapso miocárdico após a reposição nutricional (Mallet, 2002). Nos dias atuais tem-se notado quadros clínicos de desnutrição de resolução complexa e correção após cirurgias bariátricas, que não evoluíram como prevista (Ferreras, Lesmes, Compés, Murillo, \& Peris, 2005).

O esgotamento alimentar extenso somada a grandes quantidades de carboidratos administrados acarreta intenso anabolismo, com resultante consumo intracelular de eletrólitos e minerais (Mallet, 2002). A hipofosfatemia é a alteração mais 
significativa da SR, mas os indivíduos podem também apresentar hipomagnesemia, hipocalemia, hiperglicemia, deficiência de tiamina, retenção hídrica, deficiências vitamínicas e edema (Marinella, 2005; Crook, Hally, \& Panteli, 2001).

A redução abrupta dos níveis séricos desses elementos pode acarretar em rabdomiólise, distúrbio leucocitário, insuficiência cardiorrespiratória, arritmias e morte (Hearing, 2004). Apesar de estudos anteriores terem salientado a hipofosfatemia intensa como variável prevalente da síndrome de realimentação, é sabido que ocorrem outras repercussões metabólicas (Crook, \& Panteli, 2005).

A SR é resultante de modificações nas vias metabólicas para obtenção de energia em resposta ao jejum. Conceitua-se inanição aguda a supressão alimentar com duração 1 a 3 dias, o que gera gasto e esgotamento das reservas de carboidratos (glicose e glicogênio) em aproximadamente 24 horas. No jejum prolongado, quando a inanição dura mais do que três dias, ocorrem alterações adaptativas que favorecem a mobilização de gordura (lipólise) e a redução na degradação de proteínas a um nível baixo (Patel, \& Sriram 2009).

Múltiplos sistemas podem ser afetados na SR, incluindo o cardiovascular, o respiratório, e hematológico, o músculo esquelético e o neurológico. Nos casos mais graves, pode ocorrer a disfunção de múltiplos órgãos e sistemas, sendo a arritmia cardíaca a principal causa de óbito (Sriram, 2010). A anorexia nervosa é uma das principais apresentações clínicas com risco de desenvolver a síndrome, no entanto, possibilidade parecida acontece com idosos desnutridos, pacientes com câncer sob quimioterapia ou em pós-operatório (Tresley, \& Sheean, 2008).

Assim, a SR voltou a chamar a atenção da comunidade científica após a introdução da nutrição parentérica total nos anos de 1970. Não é uma síndrome rara, podendo ser observada em 0, 8 \% dos adultos hospitalizados e, em doentes oncológicos desnutridos a incidência pode atingir os $25 \%$ (Khardori, 2005). O tratamento é composto pela abordagem nutricional em caráter conservador, embora nenhuma técnica tenha sido descrita como superior às outras (Carvalho, Coelho, Rezende, \& Rocha, 2010). Considerando o impacto da SR para os pacientes, justifica-se o presente estudo. Assim, busca-se descrever a síndrome da realimentação com enfoque nos fatores de risco e diagnósticos.

\section{Metodologia}

Trata-se de um estudo de revisão integrativa de literatura. No bojo da realização da revisão, seguiu-se seis passou inter relacionados, descritos a seguir: 1) elaboração da pergunta norteadora; 2) busca na literatura; 3) coleta de dados; 4) análise crítica dos estudos incluídos; 5) discussão dos resultados e 6) apresentação da revisão integrativa (URSI ES, 2005).

Considerou-se como pergunta norteadora: Como ocorre a síndrome de realimentação? O levantamento das publicações foi realizado entre os meses de maio a julho de 2021, a partir das bases de dados LILACS, Scielo e Biblioteca Virtual em Saúde (BVS). Para busca e extração dos dados dos artigos foi utilizado um instrumento elaborado e validado para revisões integrativas de literatura com os seguintes núcleos de análise: dados de identificação do artigo (título, autores, nome do periódico, ano de publicação, volume e número), tipo de estudo, local de estudo, objetivo do estudo, eixo temático, classificação Qualis/Capes, periódico de publicação, nível de evidência, desfechos, dentre outros. A seleção foi conduzida de forma independente pelos autores, sendo as divergências solucionadas com auxílio do instrumento validado (Ursi, 2005).

Para seleção dos artigos e publicações utilizou-se os descritores, recuperados no site descritores em ciências da saúde: Síndrome da realimentação; Desnutrição energética; Desnutrição, utilizando o operador booleano "and" para combinação. Assim, foram identificados inicialmente, 60 publicações elegíveis para inclusão nesta revisão.

Para selecionar as publicações foram estabelecidos critérios de inclusão: (1) artigos disponibilizados nos respectivos bancos eletrônicos Biblioteca Virtual em Saúde (BVS), Literatura Latino-Americana e do Caribe em Ciências da Saúde (LILACS) e Scientific Electronic Library Online (Scielo), PUBMED e MEDLINE, (2) trabalhos originalmente na língua portuguesa e 
inglesa (3) materiais e publicações do Ministério da saúde do Brasil, disponível online, consoante ao propósito do presente estudo de revisar a temática síndrome de realimentação, bem como os fatores de risco e o diagnóstico no decorrer do referido corte temporal e em ordem nacional e internacional e que se firmam como importantes referências na conduta profissional no âmbito coletivo e individual.

Foram excluídos estudos que não atendiam os critérios de inclusão elencados e aqueles que não faziam uma abordagem direta a temática, resultando em 38 publicações selecionadas, por atenderem os critérios de inclusão.

\section{Resultados e Discussão}

A má nutrição hospitalar é um problema com elevada prevalência. Desde os anos trinta do século XX que foi determinada a correlação entre a perda ponderal e a morbidade e a mortalidade pós-operatória, sendo considerado importante corrigir a má nutrição para minimizar possíveis consequências (Chung, 2002; Walsh, 2001).

Frente à desnutrição grave, geralmente ocorre ansiedade por parte dos profissionais de saúde em reiniciar de forma rápida a dieta. Entretanto, a oferta intensa possui grande letalidade e a correção não adequada do déficit nutricional pode contribuir para complicações graves. Complicações que por sua vez podem conduzir à morte - síndrome de realimentação, que tem início às suas manifestações clínicas quando o carboidrato é reintroduzido (Tresley, \& Sheean, 2008; Carvalho, Coito, Lebre, Gonçalves, \& Gíria, 2008). Nesse sentido, a Síndrome de Realimentação é caracterizada por manifestações que estão diretamente relacionadas por uma disfunção eletrolítica (Melo, Sória, Tavares, \& Oliveira, 2013).

A rápida alteração do metabolismo de gorduras para o de carboidratos acarreta uma elevação significativa na disponibilidade de insulina por meio de sua maior produção. O intenso anabolismo estimula o consumo intracelular de fosfato, magnésio e potássio, produzindo diminuição súbita dos níveis séricos desses eletrólitos (Tresley, \& Sheean, 2008).

Ademais, a introdução abrupta de glicídios pode reduzir a eliminação de sódio e água e acarretar o aumento do líquido do compartimento extracelular, com processo de sobrecarga hídrica, edema no pulmão e/ou descompressão miocárdica. Outras manifestações clínicas que podem ser encontradas nesse contexto são a hiperglicemia e a deficiência de tiamina (Tresley, \& Sheean, 2008).

Esses achados também foram retratados por Stanga, Brunner, Leuenberger e Grimble (2008), na qual indicam que durante a realimentação, a disponibilidade de hidratos de carbono acarreta a elevação da insulina sanguínea e redução da secreção do glucagon. A insulina favorece a produção proteica e de glicogênio favorecendo a receptividade celular de glicose e alguns minerais, como o magnésio, fósforo, potássio, além de cofatores e a tiamina (Stanga, Brunner, Leuenberger, Grimbleet, Shenkin, Allison, \& Lobo, 2008).

Outro efeito da insulina é a elevação da reabsorção renal de sódio e água. Esse efeito é fundamental para a construção de novos tecidos e forma a recuperação do estado energético e nutricional, no entanto, o efeito metabólico deve ser controlado com o objetivo de erradicar os efeitos negativos acarretados pelo intenso influxo desses elementos para o meio intracelular e consequente redução de seus níveis séricos (Stanga, Brunner, Leuenberger, Grimbleet, Shenkin, Allison, Lobo, 2008).

Assim, a introdução da dieta pode ser encarada pelo organismo como um "elemento estressor". Reações enzimáticas inativas por longos períodos são abruptamente ativadas com a chegada de macronutrientes ao indivíduo, dando início a um cascata de reações químicas. Essa situação clínica exige uso de intensa quantidade de micronutrientes, que já são incipientes, o que acarreta uma queda adicional e em muitos casos graves, dos níveis séricos e intracelulares dos eletrólitos, além dos oligoelementos e vitaminas, resultando em uma série de manifestações clínicas em relação a deficiência de cada micronutriente e a ausência de reações enzimáticas deles dependentes (Sriram, 2010).

Dessa forma, o resultado final pode ser arritmias, convulsões, tetania, rabdomiólise, colapso miocárdio, insuficiência 
do aparelho respiratório e até mesmo morte. Esse processo acontece, frequentemente, cerca de 4 dias após o início da realimentação (Hearing, 2004).

A síndrome de realimentação afeta pacientes que são alimentados de forma intensiva por via oral, enteral ou parenteral após um grande período de nutrição deficitária e são os mais propensos a apresentar essa síndrome. De forma geral, apresentam maiores risco os pacientes com perda de peso igual ou superior a 10\% em dois a três meses ou pacientes com menos de $70 \%$ do peso corpóreo considerado adequado (Carvalho, Coito, Lebre, Gonçalves \& Gíria, 2008).

Variadas situações influenciam para a ocorrência de SR sendo elas os transtornos psiquiátricos, tais como, anorexia nervosa, alcoolismo crônico e depressão), subnutrição relacionada à dificuldade para deglutir, má absorção, jejum intenso e a hiperêmese gravídica. Outros fatores relacionados são os efeitos digestivos adversos da quimioterapia, síndrome do intestino curto, pós-operatório de cirurgia bariátrica e quadros de diabetes descompensados (Boateng, \& Sriram, 2010; Crook, 2015; National Institute for Health and Clinical Excellence, 2006).

É também observado em indivíduos com quadro clínico de marasmo e kwashiorkor; em indivíduos em jejum por período de sete a dez dias, na ocorrência de estresse e depleção; intensa perda de peso em indivíduos obesos, idosos subnutridos em realimentação; indivíduos em pós-operatório de cirurgias de grande porte; intensa repleção intravenosa de fluidos e transtornos alimentares (Crook, Hally, \& Panteli, 2001; Campos-Ferrer, Cervera-Montes, Romero, Borrás, Gómez, \& Ricart, 2004; Flesher, Archer, Leslie, McCollom, \& Martinka, 2005; Frostad, 2004; Hearing, 2004).

Estudos realizados por Kirby, Weinsier e Krundieck identificaram que a síndrome ocorre na realimentação após jejum superior a 7 dias, em associação a condições diversas, como deficiências nutricionais prévias, período pré-operatório de cirurgias de grande porte, diabetes mellitus descompensado, quimioterapia, nutrição parenteral sem adequada administração de vitaminas ou minerais, administração de aporte energético excessivo e, também, na falta de monitoramento e reposição adequada de eletrólitos (Kirby, 1995; Weinsier, \& Krundieck, 1981).

É essencial reforçar-se que a SR pode acontecer ainda nos pacientes que aparentam eutrofismo ou até mesmo nos pacientes que apresentam obesidade, conforme incidam outras variaveis de risco para a SR, conforme o quadro 1 (National Institute for Health and Clinical Excellence, 2009).

Tabela 1 - Fatores de risco para a síndrome de retroalimentação em adultos.

\section{Pacientes com risco moderado}

- Baixíssima ou nenhuma ingestão alimentar por 5 dias.

\section{Pacientes com alto risco:}

Apresentar um ou mais dos seguintes fatores de risco

- IMC menor do que $16 \mathrm{~kg} / \mathrm{m} 2$;

- Perda de peso não intencional maior ou igual a 15\% nos últimos 3 a 6 meses;

- Baixíssima ou nenhuma ingestão alimentar por pelo menos 10 dias;

- Baixos níveis de potássio, magnésio ou fósforo antes da realimentação

Ou dois ou mais dos seguintes:

- IMC menor do que $18,5 \mathrm{~kg} / \mathrm{m} 2$

- Perda de peso não intencional maior ou igual a 10\% nos últimos 3 a 6 meses;

- Baixíssima ou nenhuma ingestão alimentar por 5 dias;

- História de abuso de álcool ou outras drogas, incluindo antiácidos, diuréticos, quimioterapia e insulina.

\section{Paciente com risco muito alto:}

- IMC menor do que $14 \mathrm{~kg} / \mathrm{m} 2$;

- Baixa ou nenhuma ingestão alimentar por pelo menos 15 dias. 
IMC: Índice de massa corpórea.

Fonte: Adaptado de National Institute for Health and Clinical Excellence (2009).

A depleção nutricional é um fator frequente em pacientes com SR. Na inanição por intenso período, a reserva de glicogênio corpóreo é esgotada, enquanto as proteínas são direcionadas para funções enzimáticas e estruturais no meio intracelular, o que acarreta o uso dos estoques de gorduras como principal fonte energética. No decorrer desse período, o volume das células hepáticas, cerebrais e fibras musculares diminuem, provavelmente em razão da junção de deficiência energética, perda de acúmulo no interior das células de macromoléculas e ainda diminuição da água no meio intracelular (National Institute for Health and Clinical Excellence, 2006).

A depleção do fósforo (P) é considerada a principal consequência da SR em que o nível plasmático do íon P pode reduzir abruptamente, causando plaquetopenia (déficit de coagulação sanguínea e deficiência da função leucocitária), disfunção neuromuscular (alterações musculoesqueléticas, convulsões, cãibras ou parestesias), comprometimento da musculatura ventilatória (hipoventilação e insuficiência respiratória), alterações psicológicas (confusão mental e, eventualmente, coma) (Sobotka, 2008;Valéria, Auad, Coelho, Gonzalez, \& Silva, 2010; Franca, \& Silva, 2006; Silva, 2010).

Os desequilíbrios metabólicos citados anteriormente produzem manifestações que refletem diretamente nos diversos sistemas, sendo eles indicadores indispensáveis para o diagnóstico e intervenção precoce na síndrome de realimentação (Melo, Sória, Tavares, \& Oliveira, 2013).

Salienta-se que a SR acontece frequentemente na prática clínica, não sendo, em muitos casos, diagnosticada de forma precoce (Carvalho, Coito, Lebre, Gonçalves, \& Gíria, 2008). Inquéritos a respeito da prevalência da SR são escassos e frequentemente variáveis, influenciando para a problemática no diagnóstico assertivo, além de muitas pesquisas terem utilizado apenas a presença de hipofosfatemia como indicativo da presença da SR (Crook, 2015).

Estudo britânico conduzido em um hospital de ensino no Reino Unido, avaliou a ocorrência de SR em 243 pacientes que faziam uso de nutrição artificial. Dessa amostra, 54\% possuíam risco para o desenvolvimento de SR e 1,2\% apresentaram o diagnóstico definitivo de SR. A pesquisa determinou como critério diagnóstico de SR a presença de três dos seguintes fatores A) alteração eletrolítica intensa (potássio sérico abaixo de 2,5mmol/L, fósforo abaixo de 0,32mmol/L e magnésio menor que 0,5mmol/L); B) edema periférico ou ocorrência de hipervolemia e C) edema pulmonar, falência miocárdica e/ou respiratória (Rio, \& Whelan, 2013).

Apesar da descrição da SR ser antiga, não há estudos randomizados e controlados sobre a forma de tratamento (Caldas, \& Alves, 2015). Pesquisas indicam que o tratamento da hipofosfatemia não é frequentemente necessário a não ser que os níveis séricos de fosfato sejam menores que $1,5 \mathrm{mg} / \mathrm{dl}$ ou que o indivíduo desenvolva sintomas. Reconhece-se que a medição do fosfato plasmático não é fiel por ser um íon que apresenta alta concentração intracelular. Assim, não guarda associação com as reservas corporais totais (Crook, Hally, \& Panteli, 2001).

A terapia nutricional é definida como o conjunto de procedimentos terapêuticos para manutenção ou recuperação do estado nutricional do paciente (Silva, 2013). Os eletrólitos devem ser administrados de forma profilática, exceto se os níveis plasmáticos prévios estiverem elevados. A quantidade a ser administrada depende do peso e das concentrações plasmáticas do paciente, contudo os valores usuais são por norma, para os primeiros três dias, de 0,5 a 0,8 mEq/kg/dia para o fósforo; 1-3 $\mathrm{mEq} / \mathrm{kg} /$ dia para o potássio e 0,3 a $0,4 \mathrm{mEq} / \mathrm{kg} /$ dia para o magnésio (Stanga, Brunner, Leuenberger, Grimbleet, Shenkin, Allison, \& Lobo, 2008).

Recomenda-se que antes do início da realimentação os desequilíbrios de caráter eletrolítico sejam corrigidos e o volume circulante ajustado. Na prática clínica, estas condutas podem retardar o reinício da alimentação, mas habitualmente podem ser completadas nas primeiras 12 a 24 horas (Bankhead, Boullata, Brantley, Corkins, Guenter, Krenitsky, Lyman, Metheny, Mueller, 
Robbins, \& Wessel; 2009; Pucci, Fontes, \& Poggetti, 2008).

A terapia nutricional proporciona benefícios comprovados no tratamento de pacientes gravemente doentes, com distúrbios do trato gastrointestinal, na diminuição da morbimortalidade e com outras doenças. Porém, é necessário conhecimentos e habilidades para a sua manipulação, como para qualquer outra terapia, pois, a mesma pode trazer complicações e riscos para os pacientes, especialmente os mais críticos e com alterações metabólicas graves, se não for realizada corretamente, exigindo assim controle rigoroso (Matsuba, \& Magnoni, 2009).

\section{Conclusão}

A síndrome de realimentação está relacionada ao suporte nutricional, seja ele oral, enteral ou parenteral, na qual é ofertado de forma inadequada a pacientes com risco de desnutrição ou severamente desnutridos. É uma doença antiga, mas que ainda necessita de estudos a serem explorados nessa área. A principal característica dessa síndrome é a hipofosfatemia, no entanto, não ocorre isoladamente. Está ainda relacionada a mudanças nos níveis séricos de cálcio, potássio e magnésio, no metabolismo de carboidratos e das vitaminas, associadas aos efeitos cardíacos, neuromusculares, hematológicos, respiratórios e gastrointestinais.

Ressalta-se, porém que, nem todos os pacientes realimentados, desenvolvem a síndrome, para isso é necessário identificar os fatores de risco e monitorá-los, de forma a diminuir sua ocorrência. $\mathrm{O}$ grupo de risco inclui os pacientes sob estado de inanição, seja este agudo ou crônico e realimentados.

Alguns fatores que também podem contribuir incluem: transtornos alimentares, subnutrição associada à disfagia, má absorção, jejum prolongado, hiperêmese, pacientes com marasmo ou kwashiorkor; em jejum por sete a dez dias; significativa perda de peso em obesos; idosos subnutridos em realimentação; pacientes pós-operatório de cirurgia bariátrica; prolongada repleção intravenosa de fluidos, efeitos adversos digestivos da quimioterapia, alcoolismo crônico, diabéticos mal controlados, entre outros.

A ausência de critério de diagnóstico bem estabelecido contribui para carência de estudos que subsidiem recomendações voltadas para o manejo desses pacientes, deixando ainda lacunas a respeito das medidas de prevenção e tratamento da Síndrome de realimentação. Assim, espera-se que essa pesquisa possa contribuir para a compreensão acerca da síndrome de retroalimentação pelos profissionais de saúde e reflita na assistência efetiva aos pacientes.

\section{Referências}

Levenson, S. M., \& Seifter, E. (1985). Inanição: respostas metabólicas e fisiológicas. In: Fischer JE. Nutrição em cirurgia. Medsi, $369-416$.

Waitzberg, D. L., Caiaffa, W. T., \& Correia, M. I. T. D. (2001). Hospital malnutrition: the Brazilian national survey (IBRANUTRI): a study of 4000 patients. Nutr, 17(7):573-80.

Schnitzker, M. A., Mattman, P. E., \& Bliss, T. L. A. (1951). A clinical study of malnutrition in Japanese prisioners of war. Ann Int Med, 35 (1):69-96.

Brozek, J., Chapman, C. B., \& Keys, A. D. (1948). Drastic food restrition. JAMA, 137(18):1569-1574.

Mallet, M. (2002). Refeeding syndrome. Age Ageing, 31:65-66.

Boateng, A. A, \& Sriram K. (2010). Refeeding syndrome: Treatment considerations based on collective analysis of literature case reports. Nutrition, 26:156167.

Ferreras, J. L. T., Lesmes, I. B, Compés, C. C., Alvarez, M. C., Murillo, A. Z., \& Peris, P. G. (2005). Refeeding syndrome. A review. Rev Clin Esp, 2:79-86. Marinella, M. A. (2005). Refeeding Syndrome and Hypophosphatemia. J Intensive Care Med, 20: 155-9 4.

Crook, M. A., Hally, V., \& Panteli, J. V. (2001). The importance of the refeeding syndrome. Nutrition, 17(6):32-37.

Hearing, S. D. (2004). Refeeding syndrome: is underdiagnosed and undertreated, but treatable. BMJ, 17328(7445):908-909. 
Crook, M. A., \& Panteli, J. V. (2005). The refeeding syndrome and hypophosphataemia in the elderly. J Intern Med, 257: $397-398$.

Patel, U., \& Sriram K. (2009). Acute respiratory failure due to refeeding syndrome and hypophosphatemia induced by hypocaloric enteral nutrition. Nutrition, 25(3):364-367.

Tresley, J., \& Sheean, P. M. (2008). Refeeding syndrome: recognition Is the key to prevention and management. J Am Diet Assoc, $108(12)$ : $2105-2108$.

Khardori, R. (2005). Refeeding Syndrome and Hypophosphatemia. J Intensive Care Med, 20:174-175.

Carvalho, A. P. O., Coelho, A. T. P., Rezende, C. D., \& Rocha, J. Z. D. (2010). Anorexia nervosa e síndrome de realimentação em adolescente: relato de caso. Rev Med Minas Gerais, 20(1): 128-130.

Chung, A. (2002). Perioperative Nutrition Support. Nutrition, 18:207-208.

Walsh, C. J. (2001). Perioperative feeding: does it reduce complications?. Colorectal Disease, 3(2), 18-22, 2001.

Carvalho, C., Coito, M., Lebre, R., Gonçalves, J., \& Gíria, J. (2008). Relembrar a síndrome de realimentação. Revista Portuguesa de Cirurgia, 5(7):23-27.

Melo, R. O. L., Sória, D. A. C., Tavares, J. M., \& Oliveira, A. C. A. (2013). Síndrome de Realimentação: Implicações de Enfermagem. R. pesq.: cuid. fundam. online, 5(5):77-81.

Stanga, Z. Brunner, A. Leuenberger, M., Grimbleet, R. F., Shenkin, A., Allison, S. P., \& Lobo, D. N. (2008). Nutrition in clinical practice-the refeeding syndrome: illustrative cases and guidelines for prevention and treatment. Eur J Clinical Nutrition, 62:687-694.

Crook, M. A. (2015). Refeeding syndrome: Problems with definition and management. Nutrition, 30:1448-1455.

National Institute for Health and Clinical Excellence. (2006). Nutrition support in adults. Clinical guideline. p.32.

Campos-Ferrer, C., Cervera-Montes, M., Romero, A., Borrás, S., Gómez, E., \& Ricart, C. (2004). Cardiogenic shock associated with inappropriate nutritional regimen: refeeding syndrome. Nutr Hosp, 19(3):175-177.

Flesher, M. E., Archer, K. A., Leslie, B. D., McCollom, R. A., \& Martinka, G. P. (2005). Assessing the metabolic and clinical consequences of early enteral feeding in the malnourished patient. JPEN, 29(2):108-117.

Frostad, S. (2004). Somatic investigation and treatment of eating disorders. Tidsskr Nor Laegeforen, 124(16):2121-2125.

Kirby, D. F. (1995). Physician Workshop, Refeeding Syndrome: background, diagnoses and manegement. Proceeding of the 19th Clinical Congress: Miami, Florida, 220-225.

Weinsier, R. L., \& Krundieck, C. L. (1981). Death resulting from overzealous total parenteral nutrition: refeeding syndrome revisted. Am J Clin Nutr, 34(3): 393-399.

National Institute for Health and Clinical Excellence. (2009). Guideline for Prevention and Management of Refeeding Syndrome in Adults. (2a ed.), NHS Foundation Trust.

Sobotka, L. (2008). Bases da Nutrição Clínica, (3a ed.), Editora Rubio.

Calixto-Lima, L., Valéria Abrahão, V., Auad, G. R. V., Coelho, S. C., Gonzalez, M. C., \& Silva, R. L. da S. (2010). Manual de Nutrição Parenteral. Editora Rubio.

Franca, C. P. N., \& Silva, A. P. M. (2006). Evitando a Síndrome de Realimentação. Ver. Brás. Nutr. Clin, 21(2): 138-43.

Silva, A. I. (2010). Prevenção da Síndrome de Realimentação em Nutrição Enteral. Trabalho de Conclusão de Curso de Pós-Graduação Latu Sensu em Nutrição Clínica. São Paulo.

Rio, A., \& Whelan, K. (2013). Occurrence of refeeding syndrome in adults started on artificial nutrition support: prospective cohort study. BMJ, 3:2000-2173.

Caldas, A. C., \& Alves, J. T. M. (2015). Síndrome de realimentação em pacientes hospitalizados: série de casos e revisão da literatura. International Journal of Nutrology, 8(2):22-29.

Silva, J. W. M. da. (2013). Síndrome de realimentação. International Journal of Nutrology, 6(1):28-35.

Bankhead, R., Boullata. J., Brantley, S., Corkins, M., Guenter, P., Krenitsky, J., Lyman, B., Metheny, N. A., Mueller, C., Robbins, S., \& Wessel, J. (2009). Board of Directors. A.S.P.E.N. Enteral Nutrition Practice Recommendations. JPEN, 33(2):122-167.

Pucci, N. D., Fontes, B., \& Poggetti, R. S. (2008). Avaliação de um esquema de realimentação utilizado após 43 dias de jejum voluntário. Rev. Nutr, 21(5):503512.

Matsuba, C. S. T., \& Magnoni, D. (2009). Enfermagem em terapia nutricional. SARVIER. 